\title{
Universal quantum processor already exists and just waits for the proper programming
}

\author{
V.M. Akulin \\ Laboratoire Aime Cotton, Orsay, France
}

\begin{abstract}
A possibility of performing the C-NOT gate operation at the ground and the first excited states of two harmonic oscillators interacting via a two-level system subject to complete control is demonstrated. The system resembles Turing machine, where the result of interaction between oscillators and the two level system is restricted to a certain fixed unitary transformation matrix, while all the control required for the implementation of the gate is provided via manipulations with the two-level system, which remains the only fully-controllable part of the entire system. Each gate operation requires a "Turing programming", - it can be realized as a series of $\gtrsim 63$ elementary unitary operations. The result shows a way how one can construct a quantum processor in a multimode microwave cavity equipped with a fully controlled two-level system, such as Josephson junction chip. Parameters of already existing experimental devises could allow one to perform up to 15 gate operations in an ensemble of about 10 qubits.
\end{abstract}

Microwave resonators equipped with a superconducting qubit based on Josephson junctions became one of the most promising experimental tools for quantum state control [1] and quantum information processing [2]. Though the Law-Eberly [3] protocol for the arbitrary quantum state engineering or certain manipulations with the Schrödinger cat states [2] could be implemented in such machines, strictly speaking, the unitary transformation group of such systems does not satisfy conditions of the Jurdjevic-Sussman theorem [4] of complete controllability, since it is non-compact containing as a subgroup the non-compact group $S U(1,1)$ of harmonic oscillator.

However, in the framework of Jaynes-Cummings model, one finds regimes where the oscillator excitation cannot exceed a trapping level, for which the Rabi transition frequency to the next photon number state multiplied by the interaction time equals to a multiple of $2 \pi$. In such a regime, a finite number of lower Fock states get singled out from the entire oscillator Hilbert spaces and form an isolated Hilbert subspace with a compact group of transformations, for which the Jurdjevic-Sussman requirements do hold and hence the complete control of evolution does become achievable. Thought this control is universal, it is a bit counter-intuitive, since it implies sequences of $N^{2}$ operation for the dimension $N$ of the Hilbert subspace with no evident logic of the action at each step - just the overall action of the sequence yilds the required untary transformation.

Here we show, that in multimode resonator interacting with a single qubit, the collection of aforesaid subspaces of each mode can form a Hilbert space for quantum computation. Such a machine is simply a multimode analog of already existing experimental devices,- it just require a proper programming in the form of a sequence of number of unitary operations per each logic gate transformation. In a sense, this resembles the Turing machine for the quantum case, where the logics gates are realized as rather long sequences of basic machine operations. The question whether or not such an approach based on just a single two-level system subject to a sequences of op- eration is preferable as compared to a system or large number of two-level quantum systems subject to simpler and intuitively apparent controls [5] [6] [7] [8], is a matter of experimental convenience.

Consider in more detail a multimode quantum microwave resonator equipped with a completely controlled two-level quantum system (qubit), which can interact with the resonator modes via the "dipole" interaction. First restrict ourselves to the case of two modes at frequencies $\omega_{1}$ and $\omega_{2}$. The Hamiltonian of the system reads

$$
\begin{aligned}
\widehat{H} & =\Delta \widehat{a} \dagger \widehat{a}-\Delta \widehat{b} \dagger \widehat{b}+\delta(t) \frac{\widehat{\sigma}_{z}}{2}+X(t) \widehat{\sigma}_{x}+Y(t) \widehat{\sigma}_{y} \\
& +\left(\Omega_{1} \widehat{a} \dagger+\Omega_{2} \widehat{b} \dagger\right) \widehat{\sigma}^{-}+\left(\Omega_{1} \widehat{a}+\Omega_{2} \widehat{b}\right) \widehat{\sigma}^{+}
\end{aligned}
$$

where $\widehat{a} \dagger, \widehat{a}, \widehat{b} \dagger \widehat{b}$ are the creation and anihilation operators of the first and the second mode, respectively, $\widehat{\sigma}_{x}=\widehat{\sigma}^{-}+\widehat{\sigma}^{+}, \widehat{\sigma}_{y}=i \widehat{\sigma}^{-}-i \widehat{\sigma}^{+}, \widehat{\sigma}_{z}=2 \widehat{\sigma}^{-} \widehat{\sigma}^{+}-1$, are Pauli matrices of the two-level system, $\Delta=\frac{\omega_{1}-\omega_{2}}{2}$, $\delta(t)=\omega(t)-\frac{\omega_{1}+\omega_{2}}{2}$, while $\Omega_{1}$ and $\Omega_{2}$ are the qubit vacuum Rabi frequencies for the first and the second mode, respectively. The controlled frequency $\omega(t)$, as well as "dipole" couplings to controlled "driving fields" $X(t)$ and $Y(t)$ allows one to exert complete control over the twolevel system. We assume

$$
\Delta \gg \Omega_{1}, \Omega_{2} .
$$

The qubit is set in resonance with modes 1 and 2 in alternation, that is $\delta(t) \equiv \pm \Delta$, for the time intervals $\tau_{1}$ and $\tau_{2}$, respectively, such that

$$
\tau_{1} \Omega_{1} \sqrt{2}=\tau_{2} \Omega_{2} \sqrt{2}=2 \pi
$$

and thus the Jaynes-Cummings trapping requirement holds for the second excited states of both mode oscillators. After each of such time intervals the state of the system experience the evolutions given by the matrices

$$
\widehat{A}=e^{-i \pi \sqrt{2}\left(\widehat{a} \dagger \widehat{\sigma}^{-}+\widehat{a} \widehat{\sigma}^{+}\right)}
$$


and

$$
\widehat{B}=e^{-i \pi \sqrt{2}\left(\widehat{b} \dagger \widehat{\sigma}^{-}+\widehat{b} \widehat{\sigma}^{+}\right)},
$$

respectively, while the non-resonant corrections to this expression are small as $\Omega / 2 \Delta$. In between of these time intervals, one of three following arbitrary qubit transformations : either

$$
\widehat{U}_{x}=\exp \left[-i \widehat{\sigma}_{x} \sigma\right]
$$

with $\sigma=\int_{0}^{\tau} X(t) d t$, or

$$
\widehat{U}_{y}=\exp \left[-i \widehat{\sigma}_{y} \sigma\right],
$$

with $\sigma=\int_{0}^{\tau} Y(t) d t$, or

$$
\widehat{U}_{z}=\exp \left[-i \widehat{\sigma}_{z} \sigma\right],
$$

with $\sigma=\int_{0}^{\tau} \frac{\delta(t)}{2} d t$ and $\Delta \gg \delta(t)$, is performed. Here $\tau$ is a duration of the time interval of these manipulations. During these intervals the oscillators experience the phase transformations

$$
\widehat{U}_{\tau}=e^{-i \tau \Delta(\widehat{a} \dagger \widehat{a}-\widehat{b}+\widehat{b})} .
$$

As one can check directly, due to the condition Eq.(3), the transformations $\widehat{A}, \widehat{B}, \widehat{U}_{x}, \widehat{U}_{y}, \widehat{U}_{z}, \widehat{U}_{\tau}$ leave invariant the Hilbert subspace $\mathcal{H}_{\{0,1\}}$ spanned by $N=8$ vectors

$$
|111\rangle|110\rangle|101\rangle|100\rangle|011\rangle|010\rangle|001\rangle|000\rangle,
$$

where the first index denotes the ground or the excited states of the qubit and the second and the third indices denote that for the second and the first modes, respectively. These unitary operators have the matrix structure

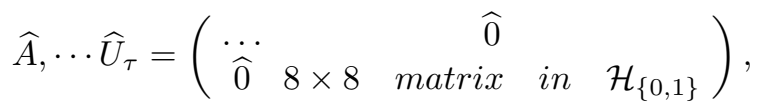

and as long as the initial state also belongs to $\mathcal{H}_{\{0,1\}}$, the operations $\widehat{A}, \widehat{B}, \widehat{U}_{x}, \widehat{U}_{y}, \widehat{U}_{z}$ and their products can be considered in this basis exclusively. The most important property of these products, is that they form a compact semigroup which contains all unitary operations in $\mathcal{H}_{\{0,1\}}$, and therefore allow one to perform in this subspace any desired unitary transformation in the form of a finite number of sequential transformations Eqs.(44). The transformations sequence should only depend on a finite number $M \geq N^{2}-1=63$ of parameters $\sigma$.

More specifically, for $\Delta \tau=2 n \pi$, where $n$ is an integer and hence $\widehat{U}_{\tau}=\widehat{1}$, a sequence

$$
\begin{gathered}
\widehat{U}_{d}=e^{-i \widehat{\sigma}_{z} \sigma_{72}} e^{-i \pi \sqrt{2}\left(\widehat{a} \dagger \widehat{\sigma}^{-}+\widehat{a} \widehat{\sigma}^{+}\right)} e^{-i \widehat{\sigma}_{y} \sigma_{71}} e^{-i \pi \sqrt{2}\left(\widehat{b} \dagger \widehat{\sigma}^{-}+\widehat{b} \widehat{\sigma}^{+}\right)} \\
e^{-i \widehat{\sigma}_{x} \sigma_{70}} e^{-i \pi \sqrt{2}\left(\widehat{a} \dagger \widehat{\sigma}^{-}+\widehat{a} \widehat{\sigma}^{+}\right)} \ldots e^{-i \widehat{\sigma}_{z} \sigma_{3}} e^{-i \pi \sqrt{2}\left(\widehat{a} \dagger \widehat{\sigma}^{-}+\widehat{a} \widehat{\sigma}^{+}\right)} \\
e^{-i \widehat{\sigma}_{y} \sigma_{2}} e^{-i \pi \sqrt{2}\left(\widehat{b} \dagger \widehat{\sigma}^{-}+\widehat{b} \widehat{\sigma}^{+}\right)} e^{-i \widehat{\sigma}_{x} \sigma_{1}} e^{-i \pi \sqrt{2}\left(\widehat{a} \dagger \widehat{\sigma}^{-}+\widehat{a} \widehat{\sigma}^{+}\right)},
\end{gathered}
$$

can be set equal to any desired $8 \times 8$ unitary matrix $\widehat{U}_{d}$ by a proper choice of the parameters $\left\{\sigma_{i}\right\}=\left\{\sigma_{M=72} \ldots \sigma_{1}\right\}$. As it has already been mentioned, there is no evident logic in the choice of the action $\sigma_{i}$ at each step $i$, only the overall result of the sequence maters. In particular, for the sequence of 72 parameters $\left\{\sigma_{i}\right\}=\{-0.2872$, $0.1842,-0.5489,0.2484,0.0132,-0.1134,-0.5642,-0.5800$, $-2.4470,-0.0432,0.3052,0.0869,0.5365,0.6245,-0.7469$, $-0.5959,-0.9621,-2.0245,-0.0107,0.3731,0.0410,0.3369$, $0.4287,0.1212,-0.6637,-0.1490,-2.5645,-0.0396,-0.0460$, $0.1488,0.2528,0.4742,0.9225,-0.3419,-0.4538,-3.4287$, $-0.2260,0.0561,0.5803,0.7112,0.8276,-0.1700,-0.1722$, $-0.6864,-2.6273,0.4602,0.2338,0.9878,0.0751,0.2090$, $-0.1949,0.1052,-0.3791,-2.4825,0.5824,0.3608,0.69429$, $1.0914,0.2271,0.2274,-0.6667,-0.1907,-3.1813,0.3526$, $-0.3946,0.2783,0.6658,0.0545,-0.4650,0.0846,-0.1140$, $-2.8158\}$ the product Eq.(11) written in the subspace basis Eq.(10) yields a transformation matrix which up to a global Berry-phase factor, is the C-NOT gate

$$
\widehat{U}_{d}=\left(\begin{array}{cccccccc}
0 & 1 & 0 & 0 & 0 & 0 & 0 & 0 \\
1 & 0 & 0 & 0 & 0 & 0 & 0 & 0 \\
0 & 0 & 1 & 0 & 0 & 0 & 0 & 0 \\
0 & 0 & 0 & 1 & 0 & 0 & 0 & 0 \\
0 & 0 & 0 & 0 & 0 & 1 & 0 & 0 \\
0 & 0 & 0 & 0 & 1 & 0 & 0 & 0 \\
0 & 0 & 0 & 0 & 0 & 0 & 1 & 0 \\
0 & 0 & 0 & 0 & 0 & 0 & 0 & 1
\end{array}\right)
$$

for the photonic part. This means that for an arbitrary initial state of the two-level system, application of the operation sequence Eq.(11) corresponds to the transformation

$$
\widehat{U}_{C-N O T}=\left(\begin{array}{llll}
0 & 1 & 0 & 0 \\
1 & 0 & 0 & 0 \\
0 & 0 & 1 & 0 \\
0 & 0 & 0 & 1
\end{array}\right)
$$

in the Hilbert subspace spanned by the ground and the first excited states of the photonic modes

$$
\{|11\rangle|10\rangle|01\rangle|00\rangle\} \text {. }
$$

The transformation Eq. (12) being a tensor product of the two-level system identity matrix and the photonic $\widehat{U}_{C-N O T}$ does not produce entanglement between the qubit and the cavity as long as the latter remains restricted to first two levels of each mode.

Many other solutions $\left\{\sigma_{i}\right\}$ can be numerically found with the procedure described in [9] 10], and each of them suits for implementation of the photonic C-NOT gate at the fundamental transitions of two modes, while the condition $\Delta \tau=2 n \pi$ can be releases just resulting in changing of the sequence $\left\{\sigma_{i}\right\}$. One should also notice, that the sequence Eq.(11) can also be build of only two out of three Pauli matrices $\widehat{\sigma}_{x, y, z}$.

It is evident, that such transformations could be applied to any other pair of the resonator modes, provided the condition Eq.(2) holds. Therefore, one can implement 
C-NOT gates to any of pair of the mode fundamental transitions thus realizing a universal quantum processor for microwave photons.

There is, however, a certain pecularity in such a processor. Typically, the two-qubit gate operation is considered as an "expensive" one, while the local operations with qubits are "cheap", that is easy to do. This is not the case for the microwave photons in the setting under consideration - the local operations with photons have also to be performed in the same 8-dimensional Hilbert subspace and therefore they also require not less than 63 control pulses $\left\{\sigma_{i}\right\}$. A practical way of doing such an operation is to first produce the swap matrix

$$
\widehat{U}_{s}=\left(\begin{array}{cccccccc}
1 & 0 & 0 & 0 & 0 & 0 & 0 & 0 \\
0 & 0 & 0 & 1 & 0 & 0 & 0 & 0 \\
0 & 0 & 1 & 0 & 0 & 0 & 0 & 0 \\
0 & 1 & 0 & 0 & 0 & 0 & 0 & 0 \\
0 & 0 & 0 & 0 & 0 & 0 & 1 & 0 \\
0 & 0 & 0 & 0 & 0 & 1 & 0 & 0 \\
0 & 0 & 0 & 0 & 1 & 0 & 0 & 0 \\
0 & 0 & 0 & 0 & 0 & 0 & 0 & 1
\end{array}\right)
$$

as a sequence o Eq. (11) with a standard set $\left\{\sigma_{i}\right\}$ of controls, say $\left\{\sigma_{i}\right\}=\{-0.3520,0.0423,-0.1621,0.4462,0.4655$ ,0.4042, 0.2449, -0.1200, -2.5231, 0.077, 0.2609, 0.7865, $0.1527,0.2210,-1.0893,0.0321,0.2538,-1.7061,-0.0262$, $0,0.2744,-0.2684,0.5115,0,0.7084,0.0365,-2.2245$, $0.5371,0.4411,0.6516,0.7463,1.2677,-0.4479,-0.4177$, $-0.3899,0.3146,0.1395,-0.3993,0.2377,0.0146,0.3367$,
0.3302, -2.6975, -0.4906, -2.4926, -0.0343, 0.0802, -0.1986, $0.6301,0.5024,0.8930,-0.2323,-0.3366,-2.7822,0.3633$, $0.3231,0.2038,0.0344,0.3335,-1.1079,-0.0373,0.1819$, $-2.3148,0.1895,-0.1227,0.4528,0,-0.3426,-0.3362$, $0.3346,-0.3548,-2.0647\}$, and than produce an arbitrary local operation with the first mode as

$$
\widehat{U}_{1}(\alpha, \beta, \gamma)=\widehat{U}_{s} e^{-i\left(\alpha \widehat{\sigma}_{x}+\beta \widehat{\sigma}_{y}+\gamma \widehat{\sigma}_{z}\right)} \widehat{U}_{s}
$$

The second mode (and the other modes) can be locally controlled by the analogy.

The most fascinating circumstance is that a device suitable for such operations has already been constructed in reality. From the detailed manuscript by K. L. Geerlings [11] one can learn that cryogenic 3D microwave resonators, which have mode frequencies in the domain of $5-15 \mathrm{GHz}$ and are equiped with a Josephson-junction qubit, can possess quality factors $Q \gtrsim 10^{5}$ and the fundamental Rabi frequencies $\Omega \sim 100 \mathrm{MHz}$. This implies that during the coherence time of the system $T \sim 100$ $\mu s e c$ a series of at least 15 gate operations each of which is a sequence of 72 unitary transformations can be implemented to set of 10 modes at a distance $2 \Delta \sim 1 \mathrm{GHz}$ one from the other. Of course, such a processor cannot yes solve the challenging problems of practical interest, such as factorization of large numbers, but it is already powerfull enough for implementation of quantum coding protocols against the coherence losses.
[1] M. Hofheinz, H. Wang, M. Ansmann, R. C. Bialczak, E. Lucero, M. Neeley, A. D. O'Connell, D. Sank, J. Wenner, J. M. Martinis, A. N. Cleland, Synthesizing arbitrary quantum states in a superconducting resonator, Nature Lett.,459,546-549, (2009) DOI: 10.1038/nature08005

[2] R. W. Heeres, P. Reinhold, N. Ofek, L. Frunzio, L. Jiang, M. H. Devoret, R. J. Schoelkopf, Implementing a universal gate set on a logical qubit encoded in an oscillator, Nature Communications | 8: 94 | DOI: 10.1038/s41467017-00045-1

[3] C. K. Law, J. H. Eberly, Arbitrary control of a quantum electromagnetic field. Phys. Rev. Lett. 76, 1055-1058 (1996) DOI: 10.1103/PhysRevLett.76.1055

[4] V. Jurdjevic, H. J. Sussman, Control Systems on Lie Groups, Theorem 7.1., Journal of Differential Equations 12, 313-329, (1972) DOI: https://doi.org/10.1016/00220396(72)90035-6

[5] A. Biswas, G. S. Agarwal, Quantum logic gates using Stark shifted Raman transitions in a cavity, Phys. Rev. A 69, 062306 (2004) DOI: https://doi.org/10.1103/PhysRevA.69.062306

[6] P. Milman , H. Ollivier , F. Yamaguchi , M. Brune, J. M. Raimond, S. Haroche, Simple quantum information algorithms in cavity QED, Jour- nal of Modern Optics, 50, 901-913 (2009) DOI: https://doi.org/10.1080/09500340308234540

[7] P. Dong, W. Song, Zh-L. Cao, Implementing Deutsch Jozsa Algorithm in Cavity QED, Commun. Theor. Phys. (Beijing, China) 46, 241-243 (2006) DOI: https://doi.org/10.1088/1674-1056/17/4/003

[8] Ch-P. Yang, S. Han, n-qubit-controlled phase gate with superconducting quantum-interference devices coupled to a resonator, Phys. Rev. A 72, 032311 (2005) DOI: https://doi.org/10.1103/PhysRevA.72.032311

[9] G. Harel, V. M. Akulin, Complete Control of Hamiltonian Quantum Systems: Engineering of Floquet Evolution, Phys. Rev. Lett. 82, 1 (1999) DOI:https://doi.org/10.1103/PhysRevLett.82.1

[10] E. Brion, F. Carlier, G. Harel, V.M. Akulin, Nonholonomic quantum control, Journal of Physics B: Atomic, Molecular and Optical Physics 44 (15), 154001 DOI: 10.1088/0953-4075/44/15/154001

[11] K. L. Geerlings, Improving Coherence of Superconducting Qubits and Resonators, Dissertation presnted at the Yale University (Director: Michel H. Devoret) (2013), short version arXiv:1204.0742 DOI: 10.1063/1.4710520 